\title{
Schlussbemerkungen der Herren Dr. Landmann, Prof. Sahli, Prof. Béraneck,
}

\section{Sehlussbemerkungen zu der vorstehenden Erwiderung des Herrn Sahli.}

\author{
Von
}

Dr. Gustar Landmann in Darmstadt.

Wenn sich Herr Sahli in seiner Erwiderung auch etwas kürzer fasst als in seiner von mir besprochenen Originalarbeit, so wird er doch weder klarer noch beweisender.

Er unterscheidet zwischen dem was er ,im allgemeinen" will und dem was er ,"zuweilen" will. „Im allgemeinen“ sucht er „die Antikörperproduktion möglichst hoch zu treiben" und noch einen Antitoxinüberschuss zu schaffen für eventuelle spätere Nachschübe. Zu dem Zweck steigert er den Patienten bis zu seinem ,individuellen Maximum", d. h. der maximalen Tuberkulindosis, welche noch ohne jede Reaktion vertragen wird. „Zuweilen“ aber sah er bessere Resultate, wenn er nur bis zu dem ,therapeutischen Optimum" ging, d. h. zu einer ,etwas niedrigeren Dosis" als das individuelle Maximum ist. Das ist der neue „Begriff" der ,immunisatorischen Heilwirkung", welche seiner Ansicht nach "toto coelo" verschieden ist von dem was bei der Immunisierung eines Diphtheriepferdes vorgeht und er behauptet, dass eine „p rinzipielle Differenz besteht zwischen Immunisierung und immunisatorischer Heilwirkung".

Ich dagegen behaupte auch jetzt noch, dass das von Herrn Sahli geschilderte Verfahren sich von dem der seither üblichen Tuberkulinbehandlung und von dem der Pferdeimmunisierung nur graduell unterscheidet und daher auch keinen neuen Namen verdient. 
Herr Sahli gibt seinen Patienten in regelmässigen Pausen steigende Dosen Tuberkulosegift um Tuberkuloseantitoxin zu erzeugen; die Diphtheriepferde erhalten in regelmässigen Pausen steigende Dosen Diphtheriegift, um Diphtherieantitoxin zu erzengen. In beiden Fällen findet das gleiche von Behring entdeckte und von Ehrlich erklärte Prinzip der Antitoxinbildung Anwendung.

Der Unterschied ist ein rein gradueller: Herr $\mathrm{Sahli}$ erzeugt mit sehr kleinen Dosen Toxin so kleine Mengen von Antitoxin, dass diese von dem im Körper des Patienten vorhandenen Toxin unter Umständen insgesamt neutralisiert werden. In dem Diphtheriepferd werden mit sehr grossen Mengen Gift so grosse Mengen Antitoxin erzeugt, dass sie - im Blutserum aufgespeichert - auf andere Individuen übertragen werden können.

Herr Sahli hat also keinen neuen Begriff, sondern überflüssigerweise nur einen neuen Namen für eine alte Sache geschaffen.

Dazu kommt noch die Inkongruenz zwischen dem, was Herr Sahli will, und dem, was er tut. Er sagt: „Wir suchen im allgemeinen die Antikörperproduktion möglichst hoch zu treiben" (S. 3) und er gibt nach 72 wöchentlicher Behandlung nur den fünfhundertsten Teil der Giftmenge, auf die Bandelier-Roepke nach 16 Wochen kommen, wohlgemerkt, ohne dass letztere „Giftiiberladung“" erhalten.

Nachdem ich so den springenden Punkt der Sahlischen Arbeit nochmals klar gestellt habe, kann ich mich darauf beschränken, die ne uen unrichtigen Behauptungen $\mathrm{Sahlis}$ zurückzuweisen.

Auf Seite 6 sagt Sabli: ,Auch erwähnt Landmann nicht, dass Béraneck in seiner zweiten Arbeit die Wirkung des Tuberkulins ebenfalls wesentlich auf Antikörperproduktion zurückführt und „so konstruiert Landmann im Interesse seiner Polemik... einen Gegensatz, der gar nicht existiert". Zu der Erwähnung der zweiten Arbeit lag für mich gar kein Grund vor, da ja diese Arbeit bei Abfassung des Sahlischen Aufsatzes, den ich besprochen habe, noch gar nicht existierte, und dass die zweite Behauptung Sahlis falsch ist, geht aus den von mir wörtlich zitierten Stellen der beiden Autoren herror.

Wenn Sahli dann sagt: „Auf diese (die experimentellen Resultate Béra necks) muss ich noch etwas näher eingehen, da sie L andmann im Interesse seines Angriffs" (muss heissen: der Abwehr des Sahlischen Angriffs d. Verf.) „teils entstellt, teils einfach verschweigt", so ist zu bemerken, dass er für die erste Behauptung den Beweis schuldig bleibt, und dass ich in meiner Arbeit, die sich in erster Linie mit der Zurückweisung des Sahlischen Angriffs auf mein Tuberkulol befasst, unmöglich auf alle "experimentellen Resultate Béranecks" 
eingehen konnte. Dazu lag um so weniger Grund vor, als Béraneck weder sein Tuberkulin concentrée, mit dem diese Resultate erzielt worden sind, noch sein Serum, das nach Sahli ,alle Arten von Antikörpern enthält", den Fachgenossen zur allgemeinen Nachprüfung zugänglich gemacht hat. Solange dies nicht geschieht, sind die „Béraneckschen Resultate“ Behauptungen, aber keine anerkannten 'Tatsachen.

Bei Besprechung der Giftigkeit der verschiedenen Tuberkuline sagt Sahli (Seite 8): „Weshalb nun Landmann, der doch auf starke Giftigkeit der Tuberkuline so grossen Wert legt, .. sich darüber aufregt, dass ich sein Tuberkulin ... als hervorragend giftig bezeichne, ist schwer verständlich und entbehrt jeder Logik". Sahli hat hier eine Abänderung seiner Worte vorgenommen, denn er hat mein Tuberkulol nicht, ,hervorragend giftig" genannt, sondern von einer ,geradezu erschreckenden Giftigkeit" gesprochen (Seite 18 seiner Broschüre). „Hervorragend giftig" würde ich natürlich als Empfehlung meines Tuberkulols betrachtet und nicht beanstandet haben, ,erschreckend" giftig aber ist eine Warnung für den Arzt, der Tuberkulol verwenden will. Wenn daher auch meine Beanstandung Herrn Sahli ,schwer verständlich" erscheint, so entbehrt sie doch durchaus nicht der Logik.

Ferner sagt Sahli: ,Es ist deshalb schwer verzeihlich, dass Landmann das Tuberkulin Béranecks durch die gänzlich aus der Luft gegriffere Behauptung zu diskreditieren sucht, das Präparat töte tuberkulöse Meerschweinchen überhaupt nicht und sei schon aus diesem Grunde wertlos". Zunächst babe ich gar nicht gesagt, das Béranecksche Tuberkulin tötet tuberkulöse Meerschweinchen überha u pt nicht, sondern (cf. S. 308, Z. 14) es tötet sie ,auch in grossen Dosen nicht". Sahli hat also hier, um seinen eigenen Ausdruck zu gebrauchen, zunächst meine Worte im Interesse seiner Polemik entstellt! Das was ich tatsächlich gesagt habe, ist nun ferner nicht aus der Luft gegriffen, sondern stützt sich auf folgende Angaben Béranecks: Auf Seite 703 seiner Arbeit sagt dieser:,.j'ai inoculé dans le péritoine de cobayes tuberculeux deux ec de mes toxines T. B. concentrées à froid dans le vide. Or, sur 4 cobayes ayant reçu ces fortes doses un seul est mort". Dies ist also die eine Komponente des Béraneckschen Tuberkulins. Von der anderen Komponente sagt Béraneck Seite 708: „A des cobayes neufs, j’ai injecté sans les tuer jusqu'à deux cc sous la peau . . Les Cobayes tuberculeux resistent dans la proportion de deux tiers environ à l'inoculation de cette forte dose". Da nun das Béranecksche T'uberkulin aus diesen fiomponenten zu gleichen Teilen besteht, so ist auch von diesem 
die für tuberkulöse Tiere sicher tödliche Dosis grösser als $2 \mathrm{ccm}$. Und da der Typus der Tuberkuline, das Kochsche, tuberkulöse Tiere in Dosen von 0,2 bis $0,5 \mathrm{ccm}$ (je nach dem Krankheitsgrad der Tiere) sicher tötet ${ }^{1}$ ), so ist meine Behauptung, das B éran ecksche 'Tuberkulin tötet auch, in grossen Dosen" tuberkulöse Tiere nicht, vollständig berechtigt und nicht ,aus der Luft gegriffen“, sondern mit Béranecks eigenen Worten bewiesen.

Ich will aber die vorstehende Zitaten aus der Béraneckschen Arbeit benützen, um mein Urteil über das Béranecksche Tuberkulin noch schärfer zu präzisieren und behaupte auf Grund der vorstehenden Angaben Béranecks: das Béranecksche Tuberkulin ist deshalb ein völlig wertvolles Präparat, weil seine für tuberkulöse Meerschweinchen sicher tödliche Dosis grösser ist als $2 \mathrm{ccm}$. Da nun schon in den $2 \mathrm{ccm}$ so viel Glyzerin und Fleischextrakt vorhanden ist, dass eventuell schon hierdurch ein tuberkulöses Meerschweinchen getötet werden kann, so darf man diese Dosis nicht mehr steigern, wenn man die Wirkung des in dem Béra neck schen Präparate vorhandenen Tuberkulosegiftes beweisen will.

Bei der Gelegenheit mache ich aber noch darauf aufmerksam, dass die stärkste Lösung Tuberkulin, welche Béraneck in den Handel bringt und von der nach $\mathrm{Sahli}$ das absolute in keinem Falle zu überschreitende Maximum 1,0 ccm beträgt, noch $62 \mathrm{mal}$ schwächer ist. Die von Sahli vorgeschlagene Maximaldosis Tuberkulin Béraneck entspricht also höchstens ${ }^{1 / 620}$ bis ${ }^{1 / 2480}$ oder durchschnittlich 1/1500 gleich $0,0006 \mathrm{ccm}$ Tuberculinum Kochii und seine Anfangsdosis $(1 / 20 \mathrm{ccm}$ Tuberkulin Béraneck A/32 cf. Sahli S. 15 und 20) entspricht 0,000000007 ccm Tuberkulinum Kochii !

Wenn nun auch Sahli an verschiedenen Stellen auf das eifrigste betont, es sei für den behandelnden Arzt ganz überflüssig, die absolute und.relative Stärke des Béraneckschen Tuberkulins zu kennen, so muss ich doch entschieden behaupten, dass der Arzt, der

1) Die unbestimmte Angabe $0,2-0,5$ beruht nicht darauf, dass das $K_{0} \mathbf{c h}$ sche Tuberkulin so stark in seiner Wirkung schwankt, sondern darauf, dass „tuberkulöses Meerschweinchen“ ja kein bestimmter Begriff ist. Der sachverständige Fachmann gibt daher aueh nie die tödliche Dosis eines Tuberkulins gegenüber einem tuberkulösen Meerschweinchen an, sondern er benützt tuberkulöse Tiere von völlig gleichem Krankheitsgrad nur daza, das zu bestimmende Tuberkulin mit einem "Standardtuberkulin" zu vergleichen (vergl. Dönitz, Untersuchungen über die Wertbestimmungen des gewöhnlichen Tuberkulins, Klin. Jahrbuch, Bd. VII). Ich musste daher, wenn ich das Tuberkulin Béranecks, für das or solche Vergleichszahlen nicht festgestellt hat, näher beleuchten wollte, die obigen Durchschnittswerte angeben. 
zwischen dem Kochschen und Béraneckschen Tuberkulin wählen soll, diese Zahlen kennen will und muss. Denn er wird es mit Recht ablehnen, sich zum gedankenlosen Werkzeug der Béraneck-Sahlischen Dosierungsvorschriften degradieren zu lassen.

Auf Seite 334 sagt Sahli: "Landmann will nicht die fundamentalen Unterschiede einsehen zwischen einem Serumtier und einem tuberkulösen Menschen." Dass diese Behauptung falsch ist, geht aus der 'Tatsache hervor, dass ich einem Serumpferde zumute, das 400000 fache der für ein gesundes Pferd noch tödlichen Dosis Diphtheriegift zu verarbeiten, dem tuberkulösen Menschen aber nur einen kleinen Bruchteil einer einzigen für einen gesunden Menschen tödlichen Dosis Tuberkulosegift.

Ganz besonders unangenebm ist Sahli offenbar meine Tabelle, in der ich die Folgen der verschiedenen Steigerungsvorschriften zahlenmässig zur Anschauung gebracht habe. Die bei der Gelegenheit von Sahli aufgeworfenen Fragen habe ich deutlich in meiner Arbeit beantwortet, seine daran geknüpften Behauptungen sind falsch. So sagt er, die zweite Dezimale könne niemand in der Injektionsspritze messen, während Frey in Davos in seiner von Sahli selbst zitierten Arbeit eine Spritze beschreibt, mit der man noch 0,025 bequem ablesen kann und die jetzt schon vielfach im Gebrauch ist. Falsch ist ferner die Behauptung $\mathrm{Sahlis}$, die Berechnung meiner "Steigerungsfaktore" sei unrichtig, denn dieselbe sei keineswegs konstant $1 / 3$ wie Landmann behaupte. Aus der Tabelle geht herror, dass meine Steigerung je $6 \mathrm{mal} 1 / 2$ und je $3 \mathrm{mal}{ }^{1 / 3}$ ist. Der Grund für die letztere Abweichung steht auf S. 311 meiner Arbeit.

Auf Seite 332 sagt Sahli: „Landmann hat vor allem die sonderbare Vorstellung, ein Tuberkulin sei um so besser, je giftiger es sei. Er stützt sich dabei auf das durchaus berechtigte Postulat der Serumexperimentatoren, für die Gewinnung eines kräftigen Immunserums auch kräftige Gifte als Antigen zu verwenden." Der zweite Satz in Verbindung mit dem folgenden dritten Satz der auf S. 325 der Sahlischen Erwiderung steht: "Wir suchen sie" (die Antikörperproduktion im Menschen bei der Tuberkulinbehandlung) „deshalb im allgemeinen möglichst hoch zu treiben " bildet eine deutliche Widerlegung des ersten Satzes. Denn wenn für ein kräftiges Immunserum, d. h. zur Frzeugung einer grossen Menge von Antikörpern ein kräftiges Gift nötig ist (Satz 2) und wenn Sahli in tuberkulösen Menschen durch die Tuberkulinbehandlung die Antikörperproduktion möglichst hoch treiben will (Satz 3), dann muss er mit mir ein kräftiges Tuberkulin für besser balten als ein schwaches, wenn er logisch 
folgern will. Die sich anschliessenden Ausführungen Shalis über die Möglichkeit einer Antikörperproduktion mit relativ ungiftigen Stoffen (Toxoiden und Toxonen) erledigen sich damit, dass man zwar auf diese Weise in vereinzelten Fällen zu wissenschaftlichen Zwecken minimale Antitoxinmengen hergestellt hat, aber niemals die Antitoxinproduktion "möglichst hochtreiben" kann, was doch Sahli erstrebt. Ebenso verhält es sich mit der Wirkung der mit Jod und anderen Chemikalien abgeschwächten Gifte, die nebenbei bemerkt, heutzutage wohl kaum noch jemand in der Serotechnik anwendet. Mit ungiftigen Substanzen und ohne Reaktionen Antitoxin erzeugen zu wollen, gleicht dem Suchen nach dem Perpetuum mobile.

Ich will nun zum Schlusse aus den zahlreichen neven Unrichtigkeiten Sahlis nur noch eine herausgreifen. Sahli erwähnt S. 344, dass meine Prioritätsansprüche Götsch gegenüber mit meinen Zahlen nicht in Einklang zu bringen seien, begründet diese Behauptung aber nicht; sie ist daher wertlos. Dann behauptet er, „dass hier keinem anderen als Lich theim die eigentliche Priorität zukommt, wie in in vielen Fragen, in welchen ihn die Literatur totgeschwiegen hat. Li c h th e im ... war der allererste Autor, welcher die reaktionslose Tuberkulinbehandlung empfahl und zwar schon gleich nach dem Bekanntwerden des 'Tuberkulins (Dentsch. Med. Wochenschr. 1901)". In der von Sahli zitierten Lichtheimschen Arbeit sagt dieser Autor bei Vorstellung einer reaktionslos behandelten Patientin auf S. 278: „Sie werden sehen, dass sie in der Heilung doch noch viel weiter zurück ist als ilrre Genossen, obwohl sie viel grössere Mengen des Giftes verbraucht hat als diese. . . Nach diesen Beobachtungen habe ich es doch für zweckmässig gehalten, von Zeit zu Zeit ordentliche Fieberreaktionen “ (v. mir gesperrt d. Verf.) „bei den Patienten herbeizuführen. Wir beginnen mit $1 \mathrm{mg}$, tritt dann keine Reaktion ein, steigen wir auf $5 \mathrm{mg}$, dann auf $1 \mathrm{cg}$. Dann lassen wir mit eintägigen Pausen die Dosen um $1 \mathrm{cg}$ steigen und genïgt auch dies nicht um ordentliche Reaktionen" (v. mir gesperrt d. Verf.) "herbeizuführen, so verlängern wir die Pausen."

Ich schliesse damit die Aufzählung neuer Sahli ischer Behauptungen, die ich als Teils den Tatsachen, teils sich selbst widersprechend nachgewiesen habe, kann aber keinen Anspruch darauf machen, das Material röllig erschöpft zu haben. Ich glaube aber, dass meine Ausführungen genügen, um dem Leser die Entscheidung darüber zu ermöglichen, ob er mir zustimmen will, der ich, wie Sahli sagt, in "naiver und elementarer" Weise die Resultate des Tierexperimentes sinngemäss auf die Tuberkulintherapie übertrage oder $\mathrm{Sahli}$, der nach seinen eigenen Worten sich nicht mit Berücksichtigung dessen begnügen will, was die klini- 
sche Beobachtung unseren fünf Sinnen zeigt, sondern „mit dem Auge des Geistes" den "Wellengang des Lebens und der Tuberkulinbehandlung" erforscht hat.

Auf die Béraneckschen Ausführungen brauche ich nicht einzugehen, da sie neues Berreismaterial gegen meine Ausführungen nicht bringen und im wesentlichen nur eine Wiederholung seiner früheren Arbeit darstellen.

\title{
Sehlussbemerkungen zu den Sehlussbemerkungen des Herrn Dr. Landmann.
}

\author{
Von
}

Prof. Dr. H. Sahli.

Herr Dr. L a ndmann gibt sich mit dem von ihm beliebten Verfahren, naturwissenschaftliche Fragen a priori entscheiden zu wollen, alle erdenkliche Mühe, den Beweis zu erbringen, dass das Tuberkulin Béraneck so wenig giftig sei, dass es sich für die Behandlung von Tuberkulösen nicht eigne. Die Pseudomathematik, durch welche Herr Landmann dasjenige was Béraneck empirisch gefunden hat, umstürzen will, mag dieser selbst zurückweisen. Ich meinerseits als Kliniker frage nur: Wie käme ich denn eigentlich, wenn das Tuberkulin Béraneck ein so ungiftiges Präparat wäre, dazu, in meiner Arbeit, die sich auf die Erfahrung stützt, fortwährend vor Giftüberlastungen $\mathrm{zu}$ warnen, und warum ist es nötig, wie jeder weiss, der selbst mit Tuberkulin Béraneck gearbeitet hat, und nicht bloss a priori spricht, wie Herr Landmann, so häufig bei empfindlichen Patienten mit den Dosen wegen ungenügender Toleranz zurückzugehen? Es kam mir noch kürzlich ein Fall zur Beobachtung, wo ein Kranker auf die Dosis von ${ }^{1 / 20} \mathrm{ccm}$ der Lösung A/128 Tuberkulin Bérane ck fieberbafte Reaktion zeigte. Es entspricht dies einer Dosis von $1 / 20$ milliontel ccm des konzentrierten Tuberkulin Béraneck und diese Menge besitzt einen Gehalt an trocknem Bakterienextrakt von bloss $1 / 20000$ milliontel ${ }^{1}$ ). Gerade diese Tatsache, dass das Tuberkulin Béraneck in so infinitesimalen Dosen bei tuberkulösen Menschen Reaktionen hervorrufen kann, dürfte in Anbetracht der experimentell festgestellten anderen Tatsache,

1) Der Exotoxingehalt des Tuberkulin Béraneck lässt sich beim Tuberkulin Béraneck ebensowenjg bestimmen wie bei anderen Tuberkulinen. 